\title{
MDROs-The New Age Challenge
}

Poonam Sharma

How to cite this article: Sharma P. MDROs-The New Age Challenge. Curr Trends Diagn Treat 2018;2(2):133-135.

Source of support: Nil

\section{Conflict of interest: None}

Dear Editor,

In continuation with our article on "Antimicrobial resistance profile in ICUs of tertiary care hospital-a worrisome state of affairs," I want to stress on alarming rise of multidrug-resistant organisms (MDROs) in our hospital. ${ }^{1}$ This study shows that $80 \%$ Enterobacteriaceae isolated from ICUs are resistant to the third-generation cephalosporins and more than $90 \%$ Staphylococcus aureus isolated are MRSA, i.e., methicillin-resistant S. aureus. A bulk of hospital-acquired infections are caused by Acinetobacter baumannii and Pseudomonas aeruginosa showing $80 \%$ of resistance to carbapenems which is much more than seen in other tertiary care hospitals. ${ }^{2}$

According to a study published in the Lancet, in 2010, India was the largest consumer of antibiotics in the world with almost 13 billion units followed by China with 10 billion units and the US with almost 7 billion units. ${ }^{3}$

Large amounts of antibiotics used for human therapy, as well as for farm animals and even for fish in aquaculture, have resulted in the selection of pathogenic bacteria resistant to multiple drugs. Experts say that if the problem of drug-resistant diseases is not tackled, it will mark a return to old days of people dying from common infections.

MDROs are defined as microorganisms, predominantly bacteria, that are resistant to one or more antimicrobial agents in three or more different classes of antimicrobials that the isolate is expected to be susceptible. ${ }^{4}$ The morbidity and mortality rates associated with MDRO infections are high. Stringent infection control strategies should be developed to prevent and control spread of MDROs and to minimize the cross infection to other patients, staff, and visitors.

Prevention and control strategies for MDROs.

A two-level approach has been recommended for the prevention and control of MDROs: ${ }^{5}$

\section{Professor}

Department of Microbiology, Sri Guru Ram Das Institute of Medical Sciences \& Research, Amritsar, Punjab, India

Corresponding Author: Poonam Sharma, Professor, Department of Microbiology, Sri Guru Ram Das Institute of Medical Sciences \& Research, Amritsar, Punjab, India, Phone: +91 9915206677, e-mail: poonam136@rediffmail.com
- Core strategies: applicable in any situation where MDRO infection or colonization is suspected or identified.

- Organism-based or resistance mechanism-based approaches: applicable if incidence or prevalence of MDROs are not decreasing despite implementation of core strategies.

\section{CORE STRATEGIES}

The implementation of transmission precautions for all patients colonized or infected with MDROs includes

- Performing hand hygiene and putting on gloves and gowns before entering the patient care area.

- Using patient-dedicated or single use noncritical patient-care equipment.

- Using a single-patient room or cohorting patients with the same strain of MDRO in designated patient care areas.

- Ensuring consistent cleaning and disinfection of surfaces in close proximity to the patient.

\section{ORGANISM-SPECIFIC APPROACH}

This approach is recommended when the incidence or prevalence of MDROs are not decreasing, despite the implementation of core strategies, and this approach focuses on the following:

- The type of MDRO (e.g., prioritization of available isolation facilities).

- The healthcare area (e.g., intensive care or other units with higher risks of transmission).

- Patient factors (e.g., whether the consequences of infection are severe).

- Available resources (e.g., feasibility of screening).

- Whether interventions to interrupt transmission are available (e.g., decolonization for MRSA).

\section{FURTHER MEASURES}

- Targeted screening: timely active screening to identify colonized patients combined with the use of contact precautions. Screening involves collecting specimens from the patient with subsequent laboratory analysis of samples. Clinicians and the infection control professionals should be informed of both positive and negative screening results promptly. 
- Decolonization: interventions may be topical, systemic, or combination of systemic and topical therapies. Topical intervention includes the use of chlorhexidine body washes and topically applied antimicrobial agents like mupirocin. Systemic intervention includes orally administered antibiotics (tetracyclines, fusidic acid, ciprofloxacin, rifampicin, and trimethoprim-sulfamethoxazole).

- Surveillance and timely feedback: increased surveillance is important to monitor the effect of interventions designed to control particular MDROs. Surveillance information should be fed back to health care workers and facility management promptly.

\section{MDRO CLEARANCE CRITERIA FOR PATIENTS}

- More than 3 months elapsed time from the last positive specimen.

- All wounds healed; no indwelling devices present.

- No exposure to any antibiotic or antiseptic body wash for at least 2 weeks prior to screening.

- In the case of MRSA, no exposure to specific antiMRSA antibiotic therapy in the past 3 months.

- Consecutive negative screens from screening sites on two separate occasions or evaluation of a single set of screening swabs with a broth amplification technique.

Some patients with vancomycin-resistant enterococcus (VRE) may appear to be clear with time but relapses with antibiotic therapy. Admission and interval screening in specialized units is an important way to detect new or relapsed VRE or MDR-GNB colonization.

Antimicrobial stewardship is the key strategy to decrease the incidence of MDROs in hospital. Good antimicrobial stewardship involves selecting an appropriate drug and optimizing the dose and duration to cure an infection while minimizing toxicity and conditions for the selection of bacterial-resistant strains. Having institution's own antibiotic policy and cumulative antibiogram are the prerequisites for antimicrobial stewardship program. The antibiotic policy depends heavily on surveillance of antimicrobial resistance (AMR) and antibiotic consumption in any setting. Therefore, it is mandatory to establish an efficient surveillance system.

Systematic surveillance at the local, regional, and global levels is a necessary tool for informing and modifying empirical antimicrobial prescription and designing and monitoring interventions to control resistance. ${ }^{6} \mathrm{WHO}$ has supported the development and release of WHONET software for routine management of data and analysis of susceptibility testing results. The software is designed to present data in a uniform manner to aid reporting and keep track of local and global resistance trends. ${ }^{7}$ WHONET is also being used worldwide for research studies. It has three main parts, a laboratory configuration file which can be used to customize it to the particular laboratory, an interface for data entry, and a part for analysis and reporting of resistance data. WHONET is an effective tool that helps not only in routine microbiology laboratory data management but also generates valuable information about antimicrobial susceptibility patterns over a place or time to provide the basis for and assess the effectiveness of prevention programs and policy decisions. The system can be implemented in SGRD hospital's microbiology laboratory. To effectively counter the threat that AMR poses, there is a need to strengthen surveillance that should provide data and monitor changes over a period and, thus, helping in controlling resistance.

WHO has recently launched the global antimicrobial resistance surveillance system (GLASS) to support a standardized approach to collection, analysis, and sharing of data on AMR at a global level. GLASS aims to combine clinical, laboratory, and epidemiological data on pathogens that pose the greatest threats to health globally. ${ }^{8}$ To streamline data collection and have nation-wide picture, the Indian Council of Medical Research (ICMR) in 2013 set up an AMR surveillance network which is currently collecting data from four tertiary care hospitals across the country through its six nodal centers on six pathogenic groups:

- Diarrheagenic bacterial organisms: CMC, Vellore

- Enteric fever pathogens: AIIMS, New Delhi

- Enterobacteriaceae causing sepsis: PGIMER, Chandigarh

- Gram-negative non-fermenters: CMC, Vellore

- Gram positives including MRSA:JIPMER, Puducherry

- Fungal infections: PGIMER, Chandigarh

The World Economic Forum in its 2013 Global Risks Report put AMR on a par with the unrestricted proliferation of weapons of mass destruction and global economic meltdown. The report points out that many people take for granted that antibiotics will always be available when we need them and that even in the highest income countries few people go throughout life without needing antibiotics. Consequently, the emergence and spread of resistance is something that should cause fear in all of us.

\section{REFERENCES}

1. Kaur N, Sharma P, Sharma S. Antimicrobial resistance profile in ICUs of a tertiary care hospital — a worrisome state of affairs. Curr Trends Diagn Treat 2018;2(1):1-3.

2. Wattal C, Goel N, Oberoi JK, et al. Surveillance of multidrug resistant organisms in tertiary care hospital in Delhi, India. J Assoc Physicians India 2010;58(Suppl):32-36

3. Van Boeckel TP, Gandra S, Ashok A, et al. Global antibiotic consumption 2000 to 2010: an analysis of national pharmaceutical sales data. Lancet Infect Dis 2014;14(8):742-750. doi: 10.1016/S1473-3099(14)70780-7. 
4. Magiorakos A, Srinivasan A, Carey RB, et al. Multidrugresistant, extensively drug-resistant and pandrug-resistant bacteria: an international expert proposal for interim standard definitions for acquired resistance. Clin Microbiol Infect 2011;18:268-281. doi: 10.1111/j.1469-0691.2011.03570.x.

5. CDC, Healthcare Infection Control Practices Advisory Committee. Guidelines for control and prevention of multidrug resistant organisms (MDROs) in Healthcare facilities, 2013. Atlanta, GA: US Department of Health and Human services, CDC, Healthcare Infection Control Practices Advisory
Committee; 2014. Available at http:/ /www.cdc.gov/ncidod / $\mathrm{dhqp} / \mathrm{pdf} / \mathrm{ar} / \mathrm{mdroguideline} 2013$

6. Halstead DC, Gomez N, McCarter YS. Reality of developing a community-wide antibiogram. J Clin Microbiol 2004;42:1-6.

7. O'Brien TF, Stelling JM. WHONET: removing obstacles to the full use of information about antibiotic resistance. Diagn Microbiol Infect Dis 1996;25:163-168.

8. Global Antimicrobial Resistance Surveillance System (GLASS). Available from: http://www.who.int/drugresistance/ surveillance/en/ 